\title{
First clinical experience with CDK4/6 inhibitors in breast cancer therapy
}

\author{
IONUT MARCEL COBEC $^{1,2}$, LAVINIA MOLERIU ${ }^{3}$, AURICA ELISABETA MOATAR $^{4,5}$ and ANDREAS REMPEN ${ }^{1}$ \\ ${ }^{1}$ Clinic of Obstetrics and Gynecology, Diakonie-Klinikum Schwäbisch Hall, D-74523 Schwäbisch Hall, Germany; \\ ${ }^{2}$ ANAPATMOL Research Center, 'Victor Babeș' University of Medicine and Pharmacy, Timișoara, 300041 Timișoara; \\ ${ }^{3}$ Department of Medical Informatics and Biostatistics, 'Victor Babeș' University of Medicine and Pharmacy, \\ Timișoara, 300041 Timișoara, Romania; ${ }^{4}$ Clinic of Internal Medicine, Hohenloher Krankenhaus Öhringen, \\ D-74613 Öhringen, Germany; ${ }^{5}$ Department of Pharmacology and Biochemistry, \\ 'Victor Babeș' University of Medicine and Pharmacy, Timișoara, 300041 Timişoara, Romania
}

Received January 8, 2021; Accepted February 9, 2021

DOI: $10.3892 /$ etm.2021.9954

\begin{abstract}
For hormone receptor-positive, HER2-negative breast cancer patients with metastatic or advanced disease, therapy with CDK4/6 inhibitors in addition to aromatase inhibitors (AIs) or to the estrogen receptor (ER) downregulator fulvestrant has resulted in an additional therapy option and a longer progression-free survival. In the Gynecologic-Oncology Clinic, Diakonie-Klinikum Schwäbisch Hall, we followed and registered our initial clinical experience with CDK4/6 inhibitors, following the side effects and tumor response over two years since they were officially approved for general use in Germany. Differences were observed when palbociclib or ribociclib was used in combination with letrozole or anastrozole or fulvestrant. The dynamic side effects and tumor response under therapy with palbociclib or ribociclib were found to be comparable with the main reported data in the official drug information. The CDK4/6 inhibitors have an important and promising role in the therapy of breast cancer patients. Patient age and therapy duration do not influence the use of palbociclib or ribociclib, although it may be important which AI is used in combination with palbociclib.
\end{abstract}

\section{Introduction}

According to the World Health Organization (WHO), breast cancer is the most frequent cancer diagnosed among women, and also accounts for the highest number of cancer-related deaths among women. In 2018, approximately $15 \%$ of all cancer-related deaths among women were attributable to

Correspondence to: Dr Ionut Marcel Cobec, Clinic of Obstetrics and Gynecology, Diakonie-Klinikum Schwäbisch Hall, 10 Diakoniestrasse, D-74523 Schwäbisch Hall, Germany

E-mail: cobec_i@yahoo.com

Key words: breast cancer, palbociclib, ribociclib, CDK4/6 inhibitors, side effects breast cancer (1). Recent research progress and newly developed therapies in the approach to breast cancer have greatly improved the quality of life and life expectancy of many patients (2).

According to the literature, $70-75 \%$ of breast cancer patient tumors express hormone receptors [estrogen receptor (ER) and/or progesterone receptor (PR) positive] $(3,4)$. As a milestone of hormone-dependent breast cancer therapy, strategies include selective ER modulators (SERMs, tamoxifen), selective ER downregulators (SERDs, fulvestrant) and estrogen biosynthesis blockers [aromatase inhibitors (AIs)] (5). More recently, hormone receptor-positive, HER2-negative breast cancer patients with metastatic or advanced disease may receive therapy with CDK4/6 inhibitors palbociclib or ribociclib in addition to AIs or to the ER downregulator fulvestrant. This additional strategy has resulted in a longer progression-free survival (PFS) compared to the antiestrogen alone treatment in patients with advanced hormone receptor-positive breast cancer (6).

The cell cycle is divided into four phases: G1, S, G2 and $M$ phase $(7,8)$. The transition from $\mathrm{Gl}$ to $\mathrm{S}$ phase is regulated by cyclin-dependent kinases (CDKs). In the presence of mitogenic signals, CDK4 and CDK6 interact with cyclin D and form the cyclin D-CDK4/6 complex which phosphorylates the retinoblastoma protein $(\mathrm{RB})$ and lifts the suppression of E2F transcription genes which causes progression from G1 to $\mathrm{S}$ phase. Activities of CDKs are affected by naturally occurring cyclin-dependent kinase inhibitors (CKIs) (9-11).

Several oncogenic pathways including the RAS-MAPK and PI3K pathways can stimulate the cyclin D-CDK4/6 complex which phosphorylates RB causing inactivation of RB leading to cell proliferation (12).

CDK4 and CDK6 together with D-type cyclins (D1, D2 and D3) promote cell cycle entry and progression through the G1 phase by inactivating RB by inhibiting an INK4 family of CDK inhibitors (CDKN2A/B). CDK4 induces cell cycle progression and also plays a key role in hormone-independent cell growth (13). A common feature of breast cancer is the aberrant activation of CDKs which leads to dysregulated cell cycle progression from $\mathrm{G} 1$ to the $\mathrm{S}$ phase. It has been shown that some cell cycle proteins, particularly those of the cyclin 
D1-CDK4/6-RB complex may play an oncogenic role in breast cancer and also as a potential therapeutic target $(13,14)$. The PALOMA-1 Study showed that the addition of palbociclib (a small-molecule inhibitor for CDK4/6) to letrozole significantly improved PFS in women with hormone receptor-positive, HER2-negative advanced breast cancer who had not received systemic treatment for advanced disease (15). Ribociclib was the second CDK4/6 inhibitor approved as treatment for hormone receptor-positive, HER2-negative advanced breast cancer in combination with an aromatase inhibitor (AI) in postmenopausal women based on the MONALESSA-2 trial (16).

Fatigue is a common side effect of palbociclib while neutropenia is a dose-limiting adverse effect of both palbociclib and ribociclib (17). Hematologic adverse events are common in palbociclib therapy, although it is associated with a higher PFS rate and lower serious complication rate (18).

Multiple fundamental studies have shown that CDK4/6 inhibitors may become a standard of care in combination with AIs or fulvestrant in women with hormone receptor-positive/HER2-negative advanced or metastatic breast cancer showing a significant increase in PFS $(12,17)$.

\section{Patients and methods}

In our gynecologic-oncology clinic of the Clinic of Obstetrics and Gynecology, Diakonie-Klinikum Schwäbisch Hall, we followed and registered our first clinical experience with palbociclib and ribociclib in the two years since palbociclib and ribociclib were officially approved for general use in Germany.

According to the drug information sheet, one cycle of therapy with palbociclib entails 28 days consisting of $1 \times 125 \mathrm{mg} /$ day per o.s. for 21 days, followed by 7 days without therapy (19).

The ribociclib drug information sheet recommends $600 \mathrm{mg}$ ribociclib/day per o.s. for 21 days, followed by 7 days without therapy; one therapy cycle has a length of 28 days (20).

For both drugs, it is recommended to administer the therapy until there is progression of the tumor or unacceptable toxicity appears.

We registered the side effects of the two drugs that were presented by the hormone receptor-positive/HER2-negative advanced or metastatic breast cancer patients receiving therapy with palbociclib or ribociclib in combination with AIs or fulvestrant. The collected data were compared with the official drug information.

Based on the clinical experience and the official drug information, the followed side effects included fatigue, leukopenia, neutropenia, thrombocytopenia, mucositis, mild alopecia, nausea, vomiting, diarrhea, elevated liver parameters (GOT, GPT and $\gamma \mathrm{GT}$ ) and atrial fibrillation. We registered also the long-term dose reduction and when during the therapy at least one week of therapy pause was necessary because of the side effects but subsequently it was possible to continue the therapy.

\section{Results}

In the two years of experience in our clinic, we followed up 24 patients $(41 \%)$ receiving therapy with palbociclib or ribociclib from a total number of 59 hormone receptor-positive/HER2-negative advanced or metastatic breast cancer patients. The other $59 \%$ were receiving another treatment or refused the therapy.

Our palbociclib/ribociclib group had a median age of 67 years with an age range of $44-87$ years. A total of $79 \%$ of our group received palbociclib and $21 \%$ received ribociclib.

In the palbociclib group, we found that the shortest administration period of palbociclib was 2 months and the longest was 34 months with ongoing therapy. In the ribociclib group, the shortest administration period was 2 months and the longest was 14 months while the patients were still receiving therapy in combination with letrozole.

Before the therapy with palbociclib was started, $79 \%$ of the group had bone metastasis, $26 \%$ had lung metastasis and $37 \%$ had liver metastasis.

Before the therapy with ribociclib was started, all patients had bone metastasis and $60 \%$ had lung metastasis. In the ribociclib group, there was no patient with liver metastasis before ribociclib was started.

In the palbociclib group $74 \%$ also received fulvestrant, $16 \%$ anastrozole and $10 \%$ letrozole.

In the ribociclib group all the patients received denosumab because of the bone metastasis and ribociclib was combined with letrozole in those cases, while in the palbociclib group just $79 \%$ were given denosumab in combination.

We documented the side effects under therapy with palbociclib (Fig. 1). The main observed and reported side effects included fatigue, leukopenia, neutropenia, thrombocytopenia, mucositis, mild alopecia, nausea, vomiting and diarrhea. In some of the patients, during the therapy with palbociclib, at least one week of therapy pause was necessary because of the side effects but subsequently it was possible to continue the therapy. However, for some of the patients long-term dose reduction was necessary.

The side effects under therapy with ribociclib (Fig. 2) consisted of leukopenia, neutropenia, thrombocytopenia, mucositis, elevated liver parameters (GOT, GPT and $\gamma \mathrm{GT}$ ), atrial fibrillation, nausea, vomiting and diarrhea. Some patients with severe side effects or persistent toxicity under therapy with ribociclib required at least a one-week therapy pause. Afterwards it was possible to continue the therapy, but in some cases long-term dose reduction was necessary.

We followed the bone metastasis under therapy with palbociclib and we noted no tumor progression in the patients where a re-staging could be done; $16 \%$ had stable disease, $47 \%$ had tumor regression and in $37 \%$ the situation was unknown because the therapy duration was too short in order to perform a re-staging.

A total of $37 \%$ of the patients with palbociclib and liver metastasis had stable disease, $5 \%$ had tumor regression, $21 \%$ had tumor progression and in $37 \%$ the situation is unknown because the therapy administration was too short for performing a re-staging.

Among the patients with lung metastasis under therapy with palbociclib, $47 \%$ showed stable disease, $11 \%$ had tumor regression, $5 \%$ had tumor progression and in $37 \%$ of the cases the situation was unknown because the therapy administration was too short for re-staging.

A total of $20 \%$ of the group under therapy with ribociclib showed stable bone metastasis and $20 \%$ stable lung metastasis 
Dynamic of the side effects under therapy with palbociclib

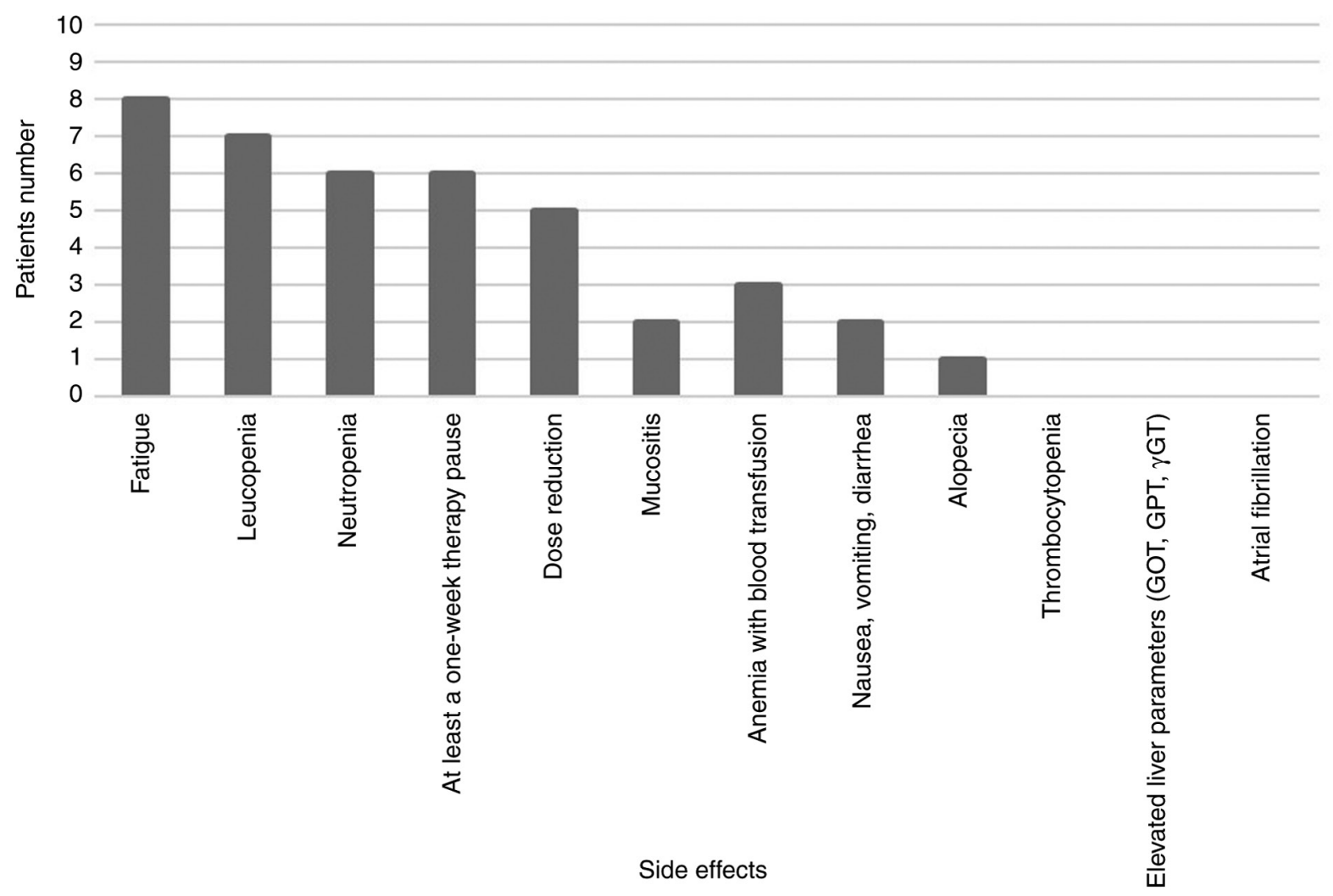

Figure 1. Dynamic of the side effects under therapy with palbociclib.

Dynamic of the side effects under therapy with ribociclib

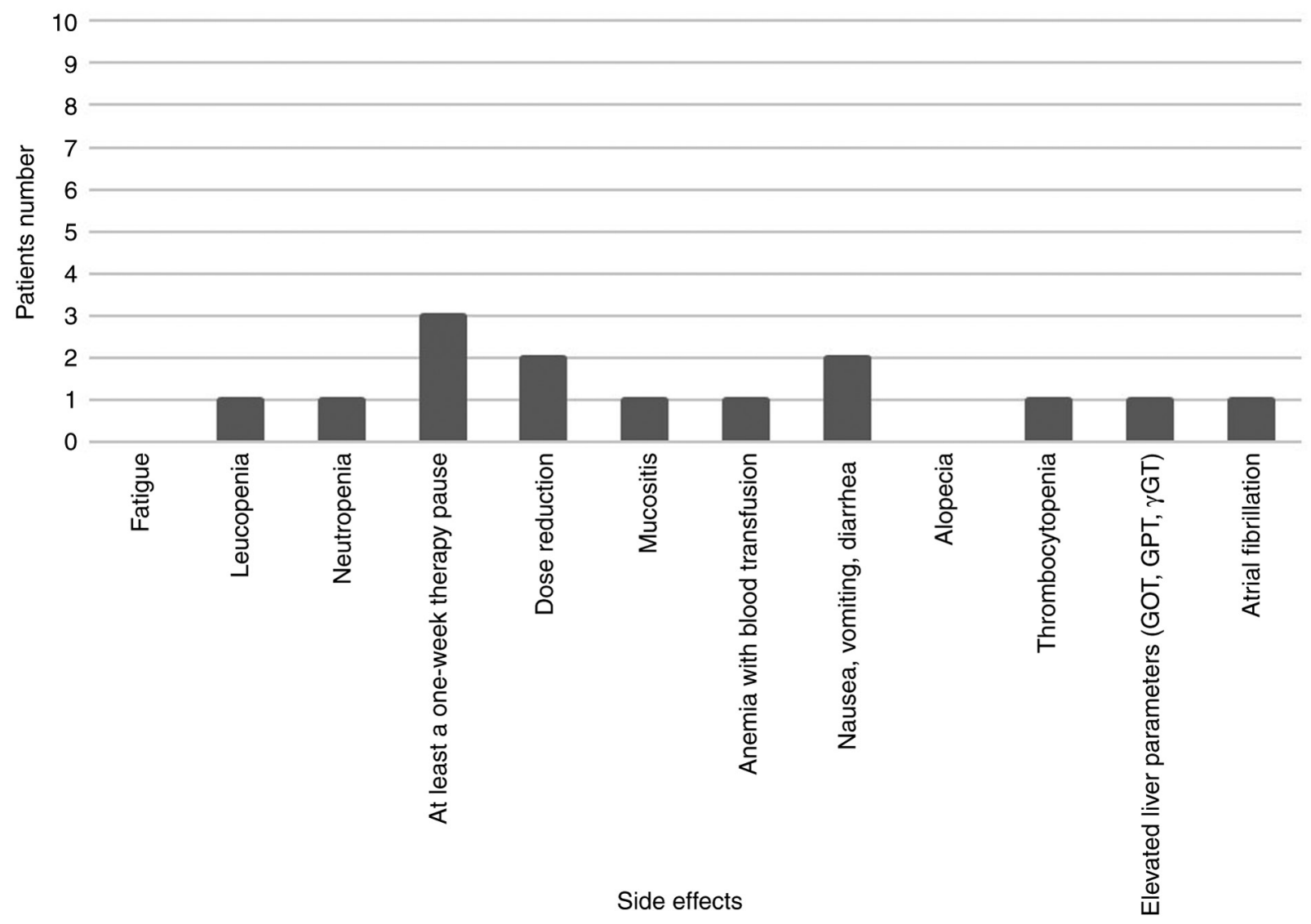

Figure 2. Dynamic of the side effects under therapy with ribociclib. 
and in $80 \%$ the situation was unknown because the therapy administration was too short for re-staging.

We noted small differences depending on whether palbociclib or ribociclib was used in combination with letrozole, anastrozole or fulvestrant.

\section{Discussion}

Both cyclin-dependent kinase (CDK)4/6 inhibitors palbociclib and ribociclib have been approved for the therapy of hormone receptor-positive/HER2-negative advanced or metastatic breast cancer in combination with aromatase inhibitors (AIs) or fulvestrant in patients who have already had endocrine therapy. In premenopausal or perimenopausal women, endocrine therapy should be combined with a luteinizing hormone-releasing hormone (LHRH) agonist (15,19-21).

The literature reports a median age of 62 and 63 years for patients under therapy with palbociclib and ribociclib depending on the performed study (15,19-21). Although our group had similar characteristics to the reported studies, we noted that our group had a median age of 67 years.

The side effects which may appear due to the possible toxicity of the two drugs are clinically relevant. A detailed medical check-up before therapy and also regular monitoring under therapy is recommended, so that the side effects are promptly identified and the patients' therapy is optimized depending on the situation (15,19-21).

According to the literature, the most frequent side effects $(\geq 20 \%$ ) of therapy with palbociclib are neutropenia, infections, leukopenia, fatigue, nausea, stomatitis, anemia, alopecia and diarrhea (19).

The side effects experienced by our patient group under therapy with palbociclib are shown in Fig. 1. The main reported complaint was fatigue followed by leukopenia, and neutropenia. A therapy pause of at least one week became necessary due to the side effects but the therapy was subsequently continued with a prolonged dose reduction performed according to the drug information. In a small number of patients, mucositis, nausea, vomiting, diarrhea, mild alopecia and thrombocytopenia were presented.

The dynamic of the side effects of ribociclib in our study (Fig. 2) revealed leukopenia, neutropenia, thrombocytopenia, mucositis, elevated liver parameters (GOT, GPT an $\gamma \mathrm{GT})$, atrial fibrillation, nausea, vomiting and diarrhea. In some patients under therapy with ribociclib, a pause of at least one week became necessary because of the side effects but the therapy was subsequently continued with a prolonged dose reduction.

In the palbociclib group, $79 \%$ had bone metastasis, $26 \%$ had lung metastasis and $37 \%$ had liver metastasis before beginning the therapy, in contrast to the palbociclib drug information which included $23.6 \%$ patients with bone metastasis at the beginning of the therapy and $49.2 \%$ visceral metastasis (19). We believe that this different distribution in metastasis is because of the different population structure. Under palbociclib-letrozole therapy a median progression-free survival (PFS) of 24.8 months has been previously reported (19). According to the PALOMA studies, the combination of palbociclib and letrozole was continued for 24 to less than 30 months, and few patients were treated for more than 36 months. A median exposure to palbociclib + endocrine therapy of 13.8 months (PALOMA-1), 19.8 months (PALOMA-2) and 10.8 months (PALOMA-3) depending on the study was reported (22). In our palbociclib group, the shortest administration period of palbociclib was 2 months and the longest was 34 months and the patient had ongoing therapy.

The ribociclib drug information reports a median therapy duration of 16.5 months and $62 \%$ of the patients received more than 12 months of therapy and a median PFS of 24.8 months for ribociclib-letrozole therapy (20). We noted in our ribociclib group that the shortest administration period was 2 months and the longest was 14 months with the patient continuing to receive the therapy in combination with letrozole.

In previous studies, the most common ( $\geq 20 \%$ ) side effects under ribociclib therapy were infections, neutropenia, leukopenia, headache, cough, nausea, fatigue, diarrhea, vomiting, constipation, alopecia, erythema and abnormal liver function tests $(20,21)$. Before the ribociclib-letrozole therapy was started, $21 \%$ of the patients had bone metastasis and $59 \%$ of the patients had visceral metastasis (20) while in our ribociclib-letrozole group before the therapy was started all patients had bone metastasis and $60 \%$ had lung metastasis. Under ribociclib-letrozole therapy a median tumor PFS interval of 25.3 months has been reported (20). In our study due to the still ongoing therapy, we did not have enough data to report on this, but the longest ribociclib-letrozole therapy administration was 14 months.

In conclusion, CDK4/6 inhibitors have an important and promising role in the therapy of the hormone receptor-positive/HER2-negative advanced or metastatic breast cancer patients. Patients age and therapy duration do not interact with the use of palbociclib or ribociclib, although it may be important which AI is used in combination with palbociclib. The longest administration period was registered in combination with letrozole.

\section{Acknowledgements}

Not applicable.

\section{Funding}

No funding was received.

\section{Availability of data and materials}

Further information concerning the present study is available from the corresponding author upon reasonable request.

\section{Authors' contributions}

IMC and AR conceived and planned in detail the present study. IMC and AEM extracted and analyzed the entire patient data. LM performed the computations and interpreted the patient data together with IMC and AEM. IMC took the lead in writing the manuscript after LM and AEM wrote some parts of the manuscript with input from AR. IMC wrote the manuscript in consultation with AR. AR supervised this study. All authors discussed the results and commented on the manuscript. All authors read and approved the final manuscript. 


\section{Ethics approval and consent to participate}

This study used pre-existing, anonymized and irreversibly de-identified data. Approval from the ethics committee was not required.

\section{Patient consent for publication}

Not applicable.

\section{Competing interests}

The authors declare that they have no competing interests.

\section{References}

1. World Health Organisation: Breast cancer. http://www.who.int/ cancer/prevention/diagnosis-screening/breast-cancer/en/. Accessed September 15, 2020

2. Ji F, Yang CQ, Li XL, Zhang LL, Yang M, Li JQ, Gao HF, Zhu T, Cheng MY, Li WP, et al: Risk of breast cancer-related death in women with a prior cancer. Aging (Albany NY) 12: 5894-5906, 2020.

3. Nadji M, Gomez-Fernandez C, Ganjei-Azar P and Morales AR: Immunohistochemistry of estrogen and progesterone receptors reconsidered: Experience with 5,993 breast cancers. Am J Clin Pathol 123: 21-27, 2005

4. Cancer Genome Atlas Network: Comprehensive molecular portraits of human breast tumours. Nature 490: 61-70, 2012.

5. Howell A, Cuzick J, Baum M, Buzdar A, Dowsett M, Forbes JF Hoctin-Boes G, Houghton J, Locker GY and Tobias JS; ATAC Trialists' Group: Results of the ATAC (arimidex, tamoxifen, alone or in combination) trial after completion of 5 years' adjuvant treatment for breast cancer. Lancet 365: 60-62, 2005.

6. Finn RS, Martin M, Rugo HS, Jones S, Im SA, Gelmon K, Harbeck N, Lipatov ON, Walshe JM, Moulder S, et al: Palbociclib and letrozole in advanced breast cancer. N Engl J Med 375: 1925-1936, 2016

7. Lundberg AS and Weinberg RA: Control of the cell cycle and apoptosis. Eur J Cancer 35: 531-539, 1999.

8. Caldon CE, Daly RJ, Sutherland RL and Musgrove EA: Cell cycle control in breast cancer cells. J Cell Biochem 97: 261-274, 2006.

9. Sherr CJ, Beach D and Shapiro GI: Targeting CDK4 and CDK6: From discovery to therapy. Cancer Discov 6: 353-367, 2016.

10. Shapiro GI: Cyclin-dependent kinase pathways as targets for cancer treatment. J Clin Oncol 24: 1770-1783, 2006.
11. Ortega S, Malumbres M and Barbacid M: Cyclin D-dependent kinases, INK4 inhibitors and cancer. Biochim Biophys Acta 1602: 73-87, 2002.

12. Gul A, Leyland-Jones B, Dey N and Pe P: A combination of the PI3K pathway inhibitor plus cell cycle pathway inhibitor to combat endocrine resistance in hormone receptor-positive breast cancer: A genomic algorithm-based treatment approach. Am J Cancer Res 8: 2359-2376, 2018.

13. Miller TW, Balko JM, Fox EM, Ghazoui Z, Dunbier A Anderson H, Dowsett M, Jiang A, Smith RA, Maira SM, et al: ER $\alpha$-dependent E2F transcription can mediate resistance to estrogen deprivation in human breast cancer. Cancer Discov 1: 338-351, 2011.

14. Finn RS, Dering J, Conklin D, Kalous O, Cohen DJ, Desai AJ, Ginther C, Atefi M, Chen I, Fowst C, et al: PD 0332991, a selective cyclin D kinase 4/6 inhibitor, preferentially inhibits proliferation of luminal estrogen receptor-positive human breast cancer cell lines in vitro. Breast Cancer Res 11: R77, 2009.

15. Finn RS, Crown JP, Lang I, Boer K, Bondarenko IM, Kulyk SO, Ettl J, Patel R, Pinter T, Schmidt M, et al: The cyclin-dependent kinase $4 / 6$ inhibitor palbociclib in combination with letrozole versus letrozole alone as first-line treatment of oestrogen receptor-positive, HER2-negative, advanced breast cancer (PALOMA-1/TRIO-18): A randomised phase 2 study. Lancet Oncol 16: 25-35, 2015.

16. Hortobagyi GN, Stemmer SM, Burris HA, Yap YS, Sonke GS, Paluch-Shimon S, Campone M, Blackwell KL, André F, Winer EP, et al: Ribociclib as first-line therapy for HR-positive, advanced breast cancer. N Engl J Med 375: 1738-1748, 2016.

17. Cersosimo RJ: Cyclin-dependent kinase $4 / 6$ inhibitors for the management of advanced or metastatic breast cancer in women. Am J Health Syst Pharm 76: 1183-1202, 2019.

18. Guo L, Hu Y, Chen X, Li Q, Wei B and Ma X: Safety and efficacy profile of cyclin-dependent kinases $4 / 6$ inhibitor palbociclib in cancer therapy: A meta-analysis of clinical trials. Cancer Med 8: 1389-1400, 2019.

19. Pfizer Inc: IBRANCE $75 / 100 / 125 \mathrm{mg}$ full prescribing information. http://labeling.pfizer.com/ShowLabeling.aspx?id=2191. Accessed September 15, 2020.

20. Novartis Pharmaceuticals Corporation: Kisqali $200 \mathrm{mg}$ prescribing information. https://www.novartis.us/sites/www. novartis.us/files/kisqali.pdf. Accessed September 15, 2020.

21. Sonke GS, Hart LL, Campone M, Erdkamp F, Janni W, Verma S, Villanueva C, Jakobsen E, Alba E, Wist E, et al: Ribociclib with letrozole vs. letrozole alone in elderly patients with hormone receptor-positive, HER2-negative breast cancer in the randomized MONALEESA-2 trial. Breast Cancer Res Treat 167: 659-669, 2018

22. Diéras V, Rugo HS, Schnell P, Gelmon K, Cristofanilli M, Loi S, Colleoni M, Lu DR, Mori A, Gauthier E, et al: Long-term pooled safety analysis of palbociclib in combination with endocrine therapy for HR+/HER2-advanced breast cancer. J Natl Cancer Inst 111: 419-430, 2019. 